\title{
Écriture et enfermement dans la poésie de Leopoldo María Panero
}

\section{Melissa Lecointre}

\section{(C) OpenEdition}

\section{Journals}

\section{Édition électronique}

URL : http://journals.openedition.org/agedor/619

DOI : 10.4000/agedor.619

ISSN : 2104-3353

Éditeur

Laboratoire LISAA

\section{Référence électronique}

Melissa Lecointre, «Écriture et enfermement dans la poésie de Leopoldo María Panero », L'Âge d'or [En ligne], 7 | 2014, mis en ligne le 01 mars 2014, consulté le 04 mai 2019. URL : http:// journals.openedition.org/agedor/619; DOI : 10.4000/agedor.619 


\title{
Melissa LECOINTRE
}

Université Paris 3 - Sorbonne Nouvelle, CREC

\section{Écriture et enfermement dans la poésie de Leopoldo María Panero}

\begin{abstract}
Résumé : La trajectoire de Leopoldo María Panero est intimement liée à sa fréquentation des hôpitaux psychiatriques dès la fin des années 1960, hôpitaux dans lesquels le poète a d'ailleurs composé une grande partie de son œuvre. Cet article étudie la manière dont l'internement a pu être à l'origine d'une poétique de l'enfermement chez un auteur qui refuse toute norme, qu'elle soit politique, sociale ou académique. L'enfermement est alors, pour le poète, une manière de se situer en dehors d'un système qu'il rejette.
\end{abstract}

Mots-clés : Leopoldo María Panero - Dissensus - Dystopie - Enfermement - Marges

Resumen: La trayectoria de Leopoldo María Panero está, desde finales de los años 60, íntimamente vinculada a estancias en hospitales psiquiátricos donde el poeta escribió la mayor parte de su obra. Este artículo estudia la manera como el internamiento ha podido influir en la elaboración de una poética del encierro en un autor que rechaza toda norma, ya sea política, social o académica. El encierro se convierte entonces para el poeta en una manera de situarse al exterior de un sistema que rechaza.

Palabras clave: Leopoldo María Panero - Disenso - Distopía - Encierro - Margen

\section{L'enfermement comme exclusion}

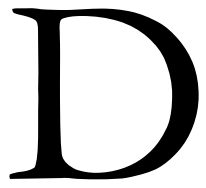

ans de nombreux entretiens, Leopoldo María Panero établit un lien fort entre enfermement et capitalisme, système qu'il perçoit comme une structure qui tente de tout contrôler: «El capitalismo es un poco como el panóptico, es como una cárcel con una torre en el centro desde donde se vigila el comportamiento de todos los presos », écrit-il en $1989^{1}$. Il ajoute que le rejet du fou et de l'ivrogne dans les sociétés sont des inventions de la bourgeoisie, qui impose ses règles et rejette ce qui va à l'encontre de son dogme. Leopoldo María Panero se réfère souvent aux écrits de Foucault pour rappeler la manière dont l'histoire a escamoté la question de la folie. Longtemps confondu avec la prison, l'asile a en effet été perçu comme un moyen d'isoler et de contenir la dangerosité, la différence, ce qui est perçu comme marginal, décalé par rapport à la norme sociale, politique et culturelle.

La trajectoire de Leopoldo María Panero est intimement liée à sa fréquentation des hôpitaux psychiatriques dès la fin des années 1960. C'est dans les hôpitaux que le poète a d'ailleurs composé la plus grande partie de son œuvre. Ses internements l'ont mené dans

${ }^{1}$ FRAILE, Eneko, «El poeta solo. Entrevista con Leopoldo María Panero », in Quimera, n 93, octobre 1989, p. 24. 
divers points de la géographie espagnole jusque dans une île, métaphore par excellence de l'enfermement, située dans les Canaries. Une mobilité comme annulée par la réitération d'un espace clos qui possède toujours les mêmes caractéristiques.

La réclusion dans la maison de santé ou dans l'asile instaure une barrière entre l'individu et le monde qui situe le poète en dehors du « système ». Les conditions d'écriture et le lieu d'où la parole poétique émerge favorisent l'apparition d'une écriture située aux marges de la société, et par là, d'un langage qui échappe à la norme et qui peut, de ce fait, participer à la création d'un dissensus. Comment l'écriture depuis un lieu cauchemardesque perçu comme une dystopie participe-t-elle à la création d'un dissensus ?

L'écriture de l'enfermement chez Leopoldo María Panero est une écriture qui se forge, en grande partie, depuis l'enferment, physique ou mental, le poète s'étant toujours senti à la marge. Mais c'est aussi une écriture qui parle de l'enfermement et qui figure cet enfermement. En situant le sujet poétique à l'extérieur du système, l'enfermement ne seraitil pas le lieu du dépassement du sensible, un moyen pour le sujet poétique de se situer «en dehors »?

Il s'agira donc de voir dans quelle mesure l'enfermement est, chez Leopoldo María Panero, une condition de l'expression d'un dissensus. Dans quelle mesure la coupure vis-àvis d'un monde et d'une Espagne qu'il rejette (que ce soit d'un point de vue politique, social et culturel), participe-t-elle à mettre à jour son dysfonctionnement et à lui substituer une nouvelle vision du réel?

\section{L'écriture depuis l'enfermement : les lieux de l'enfermement ou l'expression d'une dystopie}

La question de l'enfermement n'est pas exclusivement liée chez Leopoldo María Panero à la fréquentation des hôpitaux psychiatriques. L'enfermement est certes géographique, mais il est aussi mental, chez un poète qui se retrouve exclu et sans territoire d'abord dans l'Espagne franquiste, puis dans l'Espagne de la transition et de la démocratie dominée par le capitalisme, une Espagne qu'il n'hésite pas à rejeter à maintes occasions. Dans le texte qui accompagne sa sélection de poèmes, qui fait office de poétique, dans l'anthologie de Castellet, Nueve novísimos poetas españoles publiée en 1970, il écrit: "Vivo dentro de la fantasía paranoica del fin del mundo y no sólo no quiero salir de ella sino que pretendo que los demás entren en ella ». Et plus loin il ajoute: "Vivo bajo la sola protección de una idea: el muro de lo absoluto es para mí una enfermedad o excepción que a todos incluye $»^{2}$. Leopoldo María conçoit ici l'existence d'un monde clôturé de murs voué à la destruction dans lequel il s'inclut, et clame sa volonté d'imposer sa vision du réel aux autres.

L'enfermement apparait comme motif littéraire très tôt dans la poésie de Leopoldo María Panero. Nous trouvons dès ses premiers poèmes des lieux clos, avec notamment des portes, limites qui séparent le dedans du dehors. Dans le texte "Primer amor 》 de Primeros poemas, le sujet poétique se trouve dans une chambre fermée dont il ne souhaite pas ouvrir la porte: No llames a mi puerta deja que el viento se lleve tus labios. Auprès de lui, un cadavre conserve en lui la chaleur des baisers. Dejadme contemplar el mundo en una lágrima, écrit le sujet poétique, enfermant à son tour le monde dans l'espace aquatique de la larme, qui convoque une isotopie de l'eau, laquelle revient avec le motif de la grotte sous-marine où le sujet

${ }^{2}$ CASTELLET, José María (éd.), Nueve novísimos poetas españoles, Barcelona, Península, 2001, p. 235. 
poétique souhaite entrer: déjadme entrar en la cueva submarina. La fin du poème revient explicitement sur l'enfermement avec le motif de la cage, dans un vers séparé du reste du poème par un blanc: Pájaros pálidos en jaulas de oro. Tout le poème ne comporte qu'un seul point final, à la fin de ce vers, comme si toutes les images étaient contenues et enfermées dans l'espace clos du poème.

L'opposition dedans / dehors parcourt de nombreux textes. Elle est déjà présente dans le recueil Asi se fundó Carnaby Street, écrit en partie depuis l'enfermement physique. On pense au court texte «El hombre que quiso viajar dentro de un coche de plástico » dans lequel, derrière la portière de la voiture miniature de la marque dinki-toys, apparait, à l'intérieur, le magicien d'Oz invitándolo a entrar, séparant l'univers de la fantaisie du réel ${ }^{4}$. On pense également à cette boîte à musique llena de valses tristes qui contient la mémoire d'une époque révolue ${ }^{5}$. De nombreux poèmes sont organisés en opposant un intérieur à un extérieur, ainsi le salon de coiffure du poème en prose XXVIII (toujours dans Aqui se fundó Carnaby street), espace oppressant saturé par le tic-tac de l'horloge et l'incessant bavardage du coiffeur, qui s'opposent à l'ouverture de la rue 6 . Dans "El retorno del hijo pródigo », prose particulièrement surréaliste, le dedans concerne l'exploration d'un appareil téléphonique dans lequel un être s'introduit como en un portal oscuro ${ }^{7}$.

Il est courant de trouver dans la poésie panérienne, notamment dans les premiers recueils, une architecture et une géographie spatiales avec des chambres, des fenêtres et des murs, autant d'éléments qui renvoient à un lieu clos dans lequel le sujet poétique se représente à la première personne, ou à travers des doubles. L'espace de la chambre est souvent dans ces textes associé à l'isolement du moi et à la liquidation d'un univers de l'enfance irrémédiablement perdu, devenu inquiétant, dans lesquels les héros ont disparu, comme dans «Unas palabras para Peter Pan»: Peter Pan no es más que un nombre, un nombre más para pronunciar a solas, con voz. queda, en la habitación a oscuras ${ }^{8}$, ou encore la chambre où le vilain petit canard attend indéfiniment le héros qui ne viendra jamais lui tendre la main ${ }^{9} .$. De même dans le poème "Schekina", de Narciso en el acorde último de las flautas, on lit he muerto y en esta belada habitación donde / ya no hay nadie, y que recorre el viento, destruyendo los libros / que tanto daño bicieran, quedan sólo debajo / de las ruinas aquellos recuerdos de absurdos juegos y cópulas $y$ de niñez desenfrenada cual / un palacio enterrado bajo el mar ${ }^{10}$.

La poésie de Leopoldo María Panero est également hantée par la présence de corridors et de passages, sortes de tunnels dans lesquels déambule le sujet poétique, comme dans le célèbre "Glosa a un epitafio ", défi adressé à la figure défunte du père, où on retrouve un couloir infini, qui emprisonne et immobilise le moi : y tus pasos repiten el eco de los míos en un largo / corredor donde / retrocedo infatigable, sin / jamás moverme ${ }^{11}$.

Si l'enfermement est un motif récurrent dans la poésie de Leopoldo María Panero, certains poèmes font également référence aux lieux d'internement de manière explicite.

\footnotetext{
3 PANERO, Leopoldo María, «Primer amor », Primeros poemas, in Poesía Completa (1970-2000), édition de Túa Blesa, Madrid, Visor, 2010, p. 28.

${ }^{4}$ PANERO, Leopoldo María, «El hombre que quiso viajar dentro de un coche de plástico ", Así se fundó Carnaby Street, in Poesía Completa (1970-2000), Ibid., p. 37.

5 PANERO, Leopoldo María, «Evocación », Ibid., p. 37.

${ }^{6}$ PANERO, Leopoldo María, XXVIII, Ibid., p. 40.

${ }^{7}$ PANERO, Leopoldo María, « El retorno del hijo pródigo », Ibid., p. 41.

8 PANERO, Leopoldo María, «Unas palabras para Peter Pan », Ibid., p. 62.

9 PANERO, Leopoldo María, « Televisor anglo mejor que la realidad », Ibid., p. 55-56.

10 PANERO, Leopoldo María, "Schekina », Narciso en el acorde último de las flautas (1979), in Poesía Completa (1970-2000), op.cit., p. 147.

11 PANERO, Leopoldo María, "Glosa a un epitafio », Ibid, p 151. Voir également le poème "Los pasos en el callejón sin salida », Ibid., p 156.
} 
C'est le cas de certaines séquences de "El canto del llanero solitario », de Teoría, qui non seulement évoquent les clés de cette porte que no se abrirá nunca ${ }^{12}$, mais mentionnent explicitement l'asile comme un lieu abritant des êtres qui errent entre la vie et la mort :

\author{
Animales de contornos mágicos \\ vide Carroll \\ (el huevo con rostro humano) the rain \\ in the plain bajo el sol de las cadenas \\ el sepulcro de Sitting Bull \\ los pájaros \\ que no existen \\ el manicomio lleno de muertos vivos \\ el manicomio lleno de muertos vivos \\ el manicomio lleno de muertos vivos \\ Estas flores son cadenas \\ y yo habito en las cadenas \\ y las cadenas son la nada \\ y la nada es la roca \\ de la que no hay retorno. ${ }^{13}$
}

Le sujet poétique surgit comme attaché, des chaînes le lient au lieu, et à l'enchaînement même des mots. La répétition des trois vers qui mentionnent l'asile résonne comme seule certitude dans un univers indéchiffrable et chaotique, mêlant différentes langues et dominé par des forces irrationnelles. L'enfermement est ici à l'origine d'une écriture de l'éclatement qui tente de repousser les limites, spatiales et culturelles, mélangeant les genres, les langues, transgressant les normes.

Le lieu clos devient alors le centre à partir duquel l'individu perçoit le monde. Depuis les marges de la société, la poésie de Leopoldo María Panero devient une écriture hors normes qui se caractérise par sa transgression et par la volonté de dynamiter les repères et les bases sur lesquels les normes sont érigées, qu'elles soient politiques, sociales, ou culturelles, comme l'a analysé Claudie Terrasson dans son article "L'œuvre poétique de Leopodo María Panero : une écriture hors-normes ", où elle montre comment la poésie de Leopoldo María Panero détruit l'institution familiale, revendique une sexualité déviante, liquide l'histoire nationale de l'Espagne. Une subversion qui s'en prend même à la langue dont elle brise la syntaxe, par le biais de procédés porteurs de silence, par l'incrustation dans la langue d'autres langues ou de signes qui rendent la lecture des textes difficile, voire impossible ${ }^{14}$. L'écriture depuis les marges permet ainsi l'apparition d'une écriture hors normes caractérisée par la violence et la transgression. La voix poétique s'en prend ainsi, sans aucune retenue, à ce qui sous-tend la société pour faire l'apologie du vide ou d'une inversion qui met en avant l'inceste, les formes transgressives de la sexualité, l'exhibition morbide, la folie. L'enfermement, géographique ou psychologique, devient alors ce «dedans », situé à l'extérieur d'un système rejeté, qui permet l'expression d'un discours qui

12 PANERO, Leopoldo María, «El canto del llanero solitario », Teoría (1973), in Poesía Completa (1970-2000), édition de Túa Blesa, Madrid, Visor, 2010, p. 90.

${ }^{13}$ Ibid., p. 88.

14 TERRASSON, Claudie, "L'œuvre poétique de Leopoldo María Panero : une écriture hors-norme », in Lieux et figures de la barbarie, CECILE - EA 40-74, Université Lille 3, 2006-2008. http://evenements.univlille3.fr/colloque-barbarie2008/séminaire 
subvertit les codes et qui s'affirme comme nouvelle perception de la réalité, en proposant une autre vision du réel.

L'enfermement désigne un monde clos et reprend de manière négative certaines caractéristiques des utopies, qui s'inscrivent géographiquement souvent dans une île pensons par exemple à celle de Thomas More. L'enfermement dans l'asile participe ainsi à la création d'une utopie à l'envers, autrement dit, d'une dystopie, une société cauchemardesque où vit une communauté avec ses propres règles, et qui s'affiche en contre-modèle de l'idéal des sociétés fondées sur les bienséances, la famille, l'Église (pour ce qui est de l'utopie franquiste) ou sur la démocratie (pour les écrits les plus contemporains). L'étymologie du terme "utopie » évoque même un «lieu qui n'existe pas », et la réclusion dans l'asile, qui rejette l'individu aux marges du système, pourrait ainsi être perçue comme un lieu nié, rejeté par la société qui l'exclut.

En relisant au plus près l'un des recueils qui affiche dès son titre l'internement dans un asile, Poemas del manicomio de Mondragón, publié en 1987, on voit la manière dont l'enfermement est bien à l'origine de la création d'un lieu cauchemardesque. En outre, l'enfermement est une dimension de la pathologie même du sujet psychotique, qui tend à projeter hors de soi une réalité psychique qui lui est insoutenable et qui, de ce fait, le persécute de l'extérieur. Ce qui caractérise le mode de fonctionnement du sujet psychotique, c'est bien la projection vers l'extérieur de phénomènes internes, projection qui provoque une coupure avec le réel et entraîne une reconstruction de ce réel.

Le recueil Poemas del manicomio de Mondragón commence par un texte en prose, signé par le poète, qui décrit une cérémonie d'autodafé : «Los libros caían sobre mi máscara (y donde había un rictus de viejo moribundo), y las palabras me azotaban y un remolino de gente gritaba contra los libros, así que los eché todos a la hoguera para que el fuego deshiciera las palabras ${ }^{15}$. La scène illustre la manière dont la poésie du sujet poétique, rejetée par la communauté, est détruite par le feu: «que ardan, pues, los libros en los jardines y en los albañales y que se quemen mis versos sin salir de mis labios ", s'exclame le poète. Le recueil s'ouvre ainsi par une sorte de renoncement à l'écriture poétique et par la mise en scène de la mort de la poésie : "caigo estático de rodillas, ante el cadáver de la poesía », lit-on à la fin.

Le premier poème du recueil juxtapose, dans l'espace clos du jardin, un tableau de fleurs, d'anges, de princesses avec des éléments qui renvoient à un univers d'effroi habité par des rats, personnifications privilégiées des internés. Le sombre jardin de l'asile est, dans la poésie de Leopoldo María Panero, un égout dans lequel la vie se putréfie, et dans lequel la mort s'abat sur les fous dans un climat d'indifférence générale ${ }^{16}$. Dans de nombreux entretiens, Panero assimile l'asile à un lieu infernal, «el infierno personificado » pour reprendre ses propres termes ${ }^{17}$. Les poèmes du recueil illustrent un univers mortuaire (on note l'importance du motif de la tombe) habité par la destruction de ce qui est vivant. Ainsi, dans «El loco al que llaman el rey », le sujet poétique se situe dans un escalier, dont on insiste sur le caractère fermé, recouvert de poissons morts sur les marches. La présence du sujet poétique cloîtré entre les murs de l'hôpital devient une condamnation à mort pour un crime non perpétré, comme dans «El loco mirando desde la puerta del jardín »:

Hombre normal que por un momento

cruzas tu vida con la del esperpento

15 PANERO, Leopoldo María, «A quien me leyere », Poemas del manicomio de Mondragón (1987), in Poesía Completa $(1970-2000) \ldots$, p. 351.

16 PANERO, Leopoldo María, «En el oscuro jardín del manicomio », Ibid, p. 355.

${ }^{17}$ FRAILE, Eneko, « El poeta solo... », op. cit., p. 26. 
has de saber que no fue por matar al pelícano

sino por nada por lo que yazgo aquí entre otros sepulcros

y que a nada sino al azar y a ninguna voluntad sagrada

de demonio o de dios debo mi ruina. ${ }^{18}$

Dans un autre poème du même recueil, le sujet poétique est penché sur une poubelle, unique horizon pour celui qui est condamné au noir et à la putréfaction :

Ven hermano, estamos los dos en el suelo

hocico contra hocico, hurgando en la basura,

cuyo calor alimenta el fin de nuestras vidas

que no saben cómo terminar, atadas

las dos a esa condena que al nacer se nos impuso

peor que el olvido y la muerte

y que rasga la puerta última cerrada. ${ }^{19}$

L'univers clos de l'enfermement à Mondragon est un univers hanté par la pourriture, et par la noirceur, un lieu sans ouverture possible. Le dernier vers du poème « $\mathrm{A}$ mi madre (reivindicación de la hermosura) » figure entre parenthèses : ( sal al jardín y verás cómo la noche nos envuelve $)^{20}$. La sortie est ici sortie dans un lieu clôturé, avec une nuit qui emprisonne. Les parenthèses indiquent, dès le titre (l'expression « revendication de la beauté » figurant entre parenthèses), l'enfermement de l'écriture.

Cet univers fermé et infernal est également celui de la violence et de la transgression, comme le montrent des poèmes irrévérencieux, comme «Himno a Satán » dans lequel le sujet poétique s'en prend à l'institution religieuse en revendiquant l'union avec Satan sous le signe de la provocation : rociaremos con vino, orina y / sangre las iglesias / regalo de los magos / y debajo del crucifijo / aullaremos ${ }^{21}$.

\section{L'enfermement dans un corps}

Dans le texte en prose qui clôt Poemas del manicomio de Mondragón, on trouve une définition de la folie comme retour à la matérialité du corps organique : «La locura se puede definir, muy brevemente, como una regresión al abismo de la visión o, en otras palabras, al cuerpo humano que ésta gobierna ${ }^{22}$. Comme le rappelle Alvaro Escobar Molina dans son ouvrage L'enfermement. Espace, temps, clôture, une des conséquences de l'enfermement est l'extrême représentation du moi en émettant l'hypothèse que : «Ce qui permettrait d'engager ce processus à l'intérieur de ces espaces serait le télescopage de l'espace et du temps, s'immobilisant et produisant un espace-miroir où l'initié plongerait

18 PANERO, Leopoldo María, «El loco mirando desde la puerta del jardín », Poemas del manicomio..., op. cit., p. 356.

19 PANERO, Leopoldo María, «El loco al que llaman el rey », Ibid., p. 357-358.

${ }^{20}$ PANERO, Leopoldo María, « A mi madre » Ibid., p. 363.

${ }^{21}$ PANERO, Leopoldo María, "Himno a Satán », Ibid., p. 365.

22 PANERO, Leopoldo María, «El fin de la psiquiatría », Ibid., p. 367. 
pour se regarder et dont l'institution profiterait pour l'assujettir » ${ }^{23}$. La poésie de Panero est une poésie du corps perçu avant tout comme matière organique. La réclusion est également un enfermement dans le corps qui, outre l'extrême représentation de celui-ci, se traduit aussi par la mise en scène de tout ce qui sort de ce corps : "sangre », "semen », « orina », « excrementos ».

L'extrême représentation du moi transparait également dans le motif du miroir qui démultiplie ce moi $^{24}$. Le miroir devient alors le lieu de la pluralité du moi, à partir duquel la fragmentation de ce moi pluriel devient visible. Dans la troisième partie du poème «Los inmortales », le sujet poétique est dédoublé dans l'exiguité de l'espace :

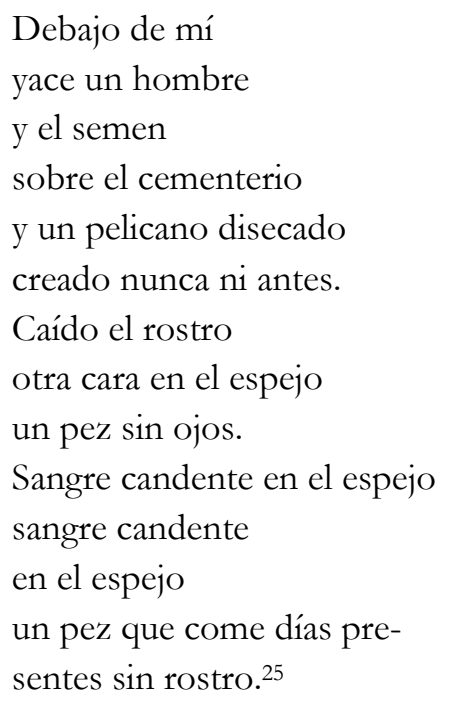

Les vers courts, les répétitions, l'enjambement lexical, qui brise l'adjectif « presente » faisant ainsi éclater l'unité de l'être, révèlent l'étroitesse de l'espace qui se referme sur le moi poétique et qui offre uniquement, à travers le retour continuel du miroir, une image du sujet poétique éclatée et méconnaissable.

\section{L'écriture de l'enfermement}

Si l'écriture de Leopoldo María Panero naît d'un enfermement, mental ou physique, cette prémisse affecte l'écriture et reste visible dans la plupart de son œuvre. L'enfermement est d'abord un emprisonnement dans le poème, avec des mots qui se heurtent sans cesse aux limites du vers. Bien que la plupart des poèmes de Panero se caractérisent par la polymétrie, la fin du vers est perçue comme une barrière infranchissable dans de nombreux textes, comme le montre l'usage très courant de l'enjambement. Le poème "Pasadizo secreto ", dans Teoría, met bien en évidence le rétrécissement de l'espace évoqué par le passage secret qui comprime les mots, par le biais de nombreux enjambements lexicaux qui tranchent la matière verbale. Le poème est composé quasi

${ }^{23}$ ESCOBAR MOLINA, Alvaro, L'enfermement. Espace, temps, clôture, Paris, Editions Klincksieck, 1989, p. 33.

${ }^{24}$ Pour le motif du miroir, voir BLESA, Túa, « El laberinto de los espejos », in Tropelías, n 1, 1990, p. 43-63.

${ }^{25}$ PANERO, Leopoldo María, «Los inmortales », Poemas del manicomio..., op. cit., p. 364. 
exclusivement de substantifs qui s'enchaînent dans une rhétorique de la répétition: Oscuridad nieve buitres desespero oscuridad nueve buitres nieve / buitres castillos (muciércalos) os / curidad nueve buitres deses / pero nieve lobos casas ${ }^{26}$. Le seul verbe conjugué est «je désespère », le reste n'étant que suite de substantifs et de paronomases qui réitèrent l'obscurité et la mort.

L'écriture de l'enfermement est une écriture de la circularité fondée sur la répétition, dans laquelle des motifs, des expressions réapparaissent, que ce soit à l'échelle du poème ou à l'échelle de l'œuvre, dévoilant une circularité dont on ne peut s'extraire, comme le voyageur de l'île qui revient toujours au même point. Túa Blesa a montré la manière dont l'œuvre de Leopoldo María Panero est truffée de références à sa propre œuvre. Ainsi, le motif de la cage d'oiseau, déjà évoqué, présent dans l'un des premiers poèmes publiés, réapparait dans le poème "La segunda esposa ", de Teoría. La reprise de ses propres vers, phénomène que l'on pourrait qualifier d'intra-textualité, figure l'enfermement du poète dans son propre monde, un monde dont il ne peut sortir. De même, la multiplicité de versions d'un même texte illustre une écriture de l'enfermement, avec sa répétition obsédante de motifs, d'images, de formulations qui apparaissent tout au long de l'œuvre, et dont Túa Blesa a montré qu'il s'agissait d'une de ses caractéristiques.

En 1997, Leolpodo María Panero quitte l’hôpital de Mondragón et sollicite l'internement dans les îles Canaries, à l'Hôpital de Gran Canaria. Le recueil Los señores del alma, de 2002, porte comme sous-titre une dédicace au psychiatre à la tête dudit hôpital : Poemas del manicomio del Dr. Rafael Inglott.

Dans ce recueil, qui revendique dans son titre une écriture forgée entre les murs, on trouve une autre esthétique pour figurer l'enfermement, également présente à d'autres moments de son œuvre. Les images closes sont remplacées par des images de vide, comme si l'enfermement se traduisait non par des espaces fermés, mais par le néant. Les portes laissent place à un lieu qui échappe à toute délimitation. La limite qui caractérise la réclusion s'efface alors pour désigner ce qui se situe au-delà du sensible. Le terme "nada » intervient dans un très grand nombre de poèmes où abondent les teintes spectrales et évanescentes, et où déambule un sujet poétique dépossédé de tout, ainsi dans les vers amor ya para nada / sólo a ruina y vejez / restos de mi figura / hombre ya para nada ${ }^{27}$. Le regard du sujet poétique se pose sur l'évanescence des choses: Oh tú, fantasma de la piedra, lit-on dans le poème consacré à Heidegger ${ }^{28}, \mathrm{O}$ tú, pálida vejez del poema, trouve-t-on au début d'un autre poème ${ }^{29}$, ou encore $O b$ tú pálida silueta del tiempo / rostro contra la nada ${ }^{30}$. Dans un monde qui s'estompe, dans lequel le langage disparaît, où le vent barre una a una las silabas, le sujet poétique erre à la recherche du néant : y que sea la nada mi último poema ${ }^{31}$. L'exaltation de la pâleur et du vide mène le poète à s'identifier lui-même, gisant, au poème, à la page : mi cadáver el único poema ${ }^{32}$.

Túa Blesa a beaucoup étudié la manière dont la poésie de Panero figure le néant et calligraphie le silence, procédés qui sont interprétés comme une manière de déconstruire le réel et d'exercer une violence sur le dire ${ }^{33}$. L'univers de ce recueil apparaît constamment

${ }^{26}$ PANERO, Leopoldo María, « Pasadizo secreto », Teoría..., op. cit., p. 115.

${ }^{27}$ PANERO, Leopodo María, «Clochard », Los señores del alma (2002), in Poesía completa (2000-2010), édition de Túa Blesa, Madrid, Visor, 2012, p. 142.

${ }^{28}$ PANERO, Leopodo María, « A Heidegger », Ibid., p. 139.

${ }^{29}$ PANERO, Leopodo María, « Segundo domingo de adviento », Ibid., p. 143.

30 PANERO, Leopodo María, « Extrapolación », Ibid., p. 144.

31 PANERO, Leopodo María « Página veinte », Ibid., p. 163.

32 PANERO, Leopodo María « Rimas », Ibid., p. 160.

33 BLESA, Túa, «El silencio, una poética del contrapoder », in Tropelías, nº11, 2000, p. 25-32 et le chapitre 3

«Caligrafías del silencio », in Leopoldo María Panero, el último poeta, Madrid, Valdemar, 1995, p. 33-45. 
dirigé vers ce néant, symbole maximal de la révolte du poète, qui ne s'exprime pas par la négation mais par le vide, expression d'un dissensus qui substitue au réel l'espace blanc.

Mais mis à part l'expression du vide, la poésie apparaît également dans ce recueil comme la possibilité non pas de dénoncer mais de retourner l'ordre en lui en imposant un autre, autre expression possible du dissensus. Le poème «Borrachera » contient le motif de la fleur inversée, que Panero reprend au poète troubadour Raimbaut d'Orange :

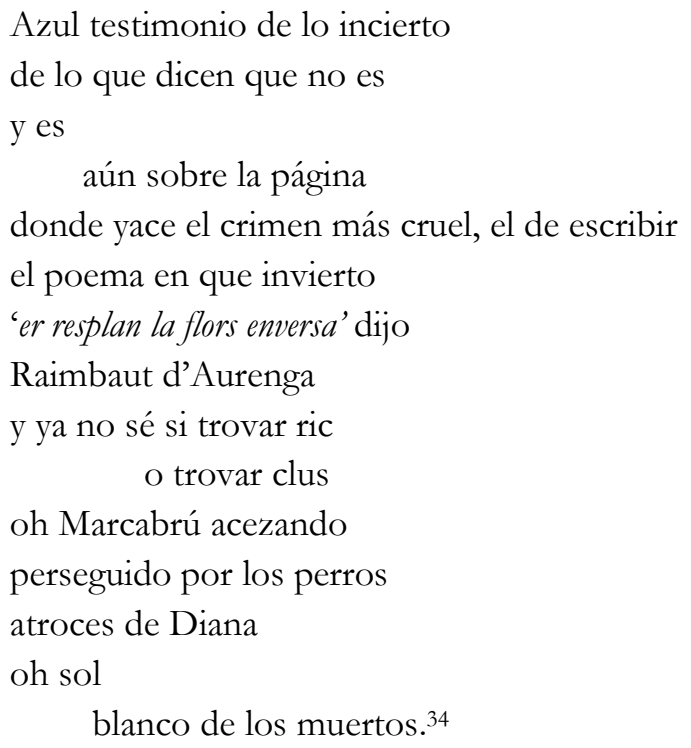

Le poème devient le lieu d'une inversion possible des choses et de l'être, d'une atteinte à la norme, ce qui rend l'écriture passible de crime.

L'inversion passe souvent dans Los señores del alma par la juxtaposition du champ lexical de l'âme et celui des excréments, du spirituel et de l'organique, de la beauté et de l'abject, comme dans "Gringo Viejo II » où les parallélismes brisent les limites entre les deux champs : ojo dorado de la mierda / ojo secreto del alma / que dibuja en el espejo mi almal ob triste espejo del hombre / que es como si mirara / una mierda a otra mierda / y creciera una flor sobre el excremento ${ }^{35}$. Tel le retournement opéré par le carnaval, la poésie de Panero exhibe ainsi ce qui est de l'ordre du bas corporel et le place au même titre que les symboles poétiques par excellence. Elle renverse la perspective en mêlant deux mondes, en instituant des rapports entre eux, et montre l'affrontement entre un système culturel classique et un système qui le transgresse. Ce recueil est également celui des interjections et d'un lyrisme qui s'affronte sans cesse à la matière organique ${ }^{36}$.

Dans cet enfermement situé hors d'un système rejeté, la poésie de Panero tend vers le dehors. Dans le confinement aux marges de la société, le langage ne peut se déployer que vers un "dehors ", dans lequel le sujet tend à disparaître pour devenir un être de langage traversé par des forces.

L'hyperprésence de citations et de références à la littérature qui habite les textes de Leopoldo María Panero ne serait-elle pas la manifestation de cette «pensée du dehors » dont parle Michel Foucault ? : «La parole de la parole nous mène par la littérature, mais

\footnotetext{
34 PANERO, Leopoldo María, «Borrachera », Los señores del..., op. cit., p. 162-163.

35 PANERO, Leopoldo María, « Gringo viejo II », Ibid., p. 144-145.

36 Voir sur ce point l'article de Túa BLESA, "'Ah' 'oh': la escritura (última) de Leopoldo María Panero », in Tránsitos: escritos sobre poesía, Valencia, Tirant lo Blanch, 2004, p. 137-140.
} 
peut-être aussi par d'autres chemins, à ce dehors où disparaît le sujet qui parle $»^{37}$. Le tissage littéraire produit par les textes de Panero situe le sujet dans une extériorité qui met en évidence l'éclatement du sujet en tant que maître du langage et des représentations. Túa Blesa a montré la manière dont la poésie de Panero, qu'il qualifie d'organique, est conçue comme une réécriture constante d'elle-même et d'autres textes de la littérature : des contes pour enfants (Peter Pan, Blanche neige), mais aussi d'auteurs comme Alfonse Daudet, Bécquer, Dashiell Hammet, Conan Doyle, Eliot, Browning, Shakespeare, Pessoa, Borges, Baudelaire, Coleridge, Mallarmé, Milton ${ }^{38}$. Ce recours constant à la tradition littéraire, qui nourrit l'œuvre de Panero et la façonne, n'est-il pas le signe d'une parole qui n'a plus de territoire et qui a perdu tout ancrage? Une parole qui pourrait se rapprocher de la description du langage établie par Foucault, d'un langage «qui a toujours commencé » et qui, pour reprendre les termes du philosophe :

doit être tourné non pas vers une confirmation intérieure, - vers une sorte de certitude centrale d'où il ne pourrait plus être délogé - mais plutôt vers une extrémité où il lui faut toujours se contester : parvenu au bord de lui-même, il ne voit pas surgir la positivité qui le contredit, mais le vide dans lequel il va s'effacer; et vers ce vide il doit aller, en acceptant de se dénouer dans la rumeur, dans l'immédiate négation de ce qu'il dit, dans un silence qui n’est pas l'intimité d'un secret mais le pur dehors où les mots se déroulent indéfiniment. ${ }^{39}$

L'enfermement chez Leopoldo María Panero pose la question du territoire comme lieu réel, mental et littéraire. Dans tous les cas, l'enfermement est, chez Panero, oxymorique : c'est un enfermement dehors. L'enfermement à l'extérieur ouvre ainsi vers un dehors dans lequel l'écriture se déploie dans une liberté totale. L'enfermement s'incarne ainsi non seulement dans des lieux clos qui recréent une sorte de dystopie ou monde négatif, mais aussi dans un néant qui montre la disparition du sujet au profit de la libération de la parole, d'une parole qui revendique son étrangeté et son extraterritorialité. L'écriture de l'enfermement, depuis les marges, permet le déploiement d'une écriture qui ne connait pas de limites. Il ne s'agit pas ici de montrer la positivité de l'enfermement, mais la manière dont, à partir de ce motif, on peut mettre à jour une expérience de dissensus qui, depuis les marges, reconfigure le centre, ordonne le réel autrement et ouvre sur d'autres espaces.

\footnotetext{
${ }^{37}$ FOUCAULT, Michel, La pensée du dehors, Montpellier, Editions Fata Morgana, 2003, p. 13-14.

38 BLESA, Túa, Leopoldo María Panero, el último..., op. cit., p. 99.

${ }^{39}$ FOUCAULT, Michel, La pensée du dehors..., op. cit., p. 21-22.
} 